Ann. Geophysicae 17, 53-65 (1999) (C EGS - Springer-Verlag 1999

\title{
Hydromagnetic spectroscopy of the magnetosphere with Pc3 geomagnetic pulsations along the $210^{\circ}$ meridian
}

\author{
V. Pilipenko ${ }^{1}$, K. Yumoto $^{2}$, E. Fedorov ${ }^{1}$, N. Yagova ${ }^{1}$ \\ ${ }^{1}$ Institute of the Earth Physics, Moscow 123810, Russia \\ ${ }^{2}$ Kyushu University, Fukuoka 812-81, Japan \\ Received: 9 September 1996 / Revised: 4 June 1998 / Accepted: 11 June 1998
}

\begin{abstract}
Analysis of Pc3 observational data along the $210^{\circ}$ magnetic meridian showed a complicated frequency-latitude structure at middle latitudes. The observed period-latitude distributions vary between events with a "noisy source": the D component has a colored-noise spectrum, while the spectrum of $H$ component exhibits regular peaks that vary with latitude, and events with a "band-limited source": the spectral power density of the $D$ component is enhanced at certain frequencies throughout the network. For most ULF events a local gap of the $H$ component amplitude has been exhibited at both conjugate stations at $L \simeq 2.1$. A quantitative interpretation has been given assuming that bandlimited MHD emission from an extra-magnetospheric source is distorted by local field line resonances. Resonant frequencies had been singled out with the use of the asymmetry between spectra of $H$ and $D$ components. Additionally, a local resonant frequency at $L \simeq 1.6$ was determined by the quasi-gradient method using the data from nearly conjugate stations. The experimentally determined local resonance frequencies agree satisfactorily with those obtained from a numerical model of the Alfven resonator with the equatorial plasma density taken by extrapolation of CarpenterAnderson model. We demonstrate how simple methods of hydromagnetic spectroscopy enable us to monitor simultaneously both the magnitude of the IMF and the magnetospheric plasma density from ULF data.
\end{abstract}

Key words. Magnetospheric physics (Magnetosphereionosphere interactions; MHD waves and instabilities; plasmasphere).

Correspondence to: V. Pilipenko

\section{Introduction}

A typical feature of the wave structure of the magnetosphere is the large variety of ULF pulsations. The wave structure of a disturbed terrestrial magnetic field may be composed by forced oscillations from external sources, harmonics of resonant Alfven field line oscillations, cavity-type oscillations of the magnetospheric volume, fluctuations of global current systems, etc. As a result, the most common Pc3-4 pulsations possess complicated spectral forms. The usual application of ULF ground data for the diagnosis of the solar wind (Russell and Fleming, 1976; Yumoto, 1985) and of the magnetosphere (Webb et al., 1977) has relied mainly on the determination of the pulsation frequency. While numerous studies have confirmed that there is strong statistical evidence for the control of Pc3-4 frequency by the magnitude of interplanetary magnetic field (IMF), other studies, including most satellite observations, have not shown such strong control (e.g., see review by Odera, 1986). However, the selection of a proper frequency can be uncertain due to complicated spectral forms of pulsations. Thus the hydromagnetic diagnosis of outer space in fact reduces to a distinct problem, which may be called a hydromagnetic spectroscopy, i.e., to the physical interpretation of spectral peaks of recorded ULF hydromagnetic emissions. Reliable interpretation of ground-based data cannot be done by just analyzing spectral features of some wave components only. The physical nature of particular spectral peaks can be revealed with the use of additional information about spatial or polarization structures of ULF signals (Green et al., 1993; Baransky et al., 1995). In this work this approach has been applied to the ULF data from mid-latitude stations along $210^{\circ}$ meridian, covering the range $L=2.9-1.5$ in the Northern and the Southern Hemispheres. 


\section{Variations of ULF spectra along a meridian}

From the network elongated along the $210^{\circ}$ meridian we have used the data of observations in SeptemberOctober 1992 from the stations: Magadan (MGD, $L=2.85$, geomagnetic longitude $\Phi=218.3^{\circ}$ ), Paratunka (PTK, $L=2.11, \Phi=225.6^{\circ}$ ), Moshiri (MSR, $\left.L=1.60, \Phi=213.0^{\circ}\right)$, in the Northern Hemisphere and Adelaide (ADL, $L=2.13, \Phi=213.3^{\circ}$ ), Birdsville (BSV, $\left.L=1.57, \Phi=212.9^{\circ}\right)$, in the Southern Hemisphere. The identical flux-gate magnetometers at each station enable us to record three-component magnetic variations between $0-0.5 \mathrm{~Hz}$ with a flat frequency response. Instrument sensitivity is about $0.1 \mathrm{nT}$ and the time resolution is $1 \mathrm{~s}$ (Yumoto et al., 1995b). For spectral and cross-spectral analysis the Blackmann-Tukey method has been used in the form described by Kurchashov et al . (1987).

The quasi-static variations, with durations of several minutes and more, are in-phase at all the stations of one hemisphere and their amplitudes decrease gradually towards the equator. In contrast with these quasi-static variations, the Pc3 ULF oscillations demonstrate a large variety of non-monotonic latitudinal distributions. A particular ULF response of the magnetosphere to an external source of wave energy with unknown spectra is determined, in our opinion, by the relationships between the spectral features of an external pulsation source and the local resonant frequencies. Depending on these relationships the seemingly different latitude-frequency distributions of Pc3 waves are observed. We now present two typical events.

\subsection{A noisy source}

The first event (1) 15.10.92, 02:58-03:08 UT is characterized by the obvious latitude dependence of spectral maximum frequency of the $H$-component and no evident spectral peaks in the $D$-component spectra. These series of Pc3 pulsations are observed under moderately disturbed geomagnetic conditions: $K_{p}=5^{\circ}$, $D_{s t}=-36 \mathrm{nT}$. Figure 1 shows band-pass filtered 10-150 $\mathrm{mHz}$ magnetograms of the $H$-component at stations from Northern (MGD, PTK, MSR) and Southern (BSV, ADL) Hemispheres. Even a visual inspection of these magnetograms reveals the essential variations of amplitude and apparent period from station to station. The significant changes of a spectral content of the $H$-component between different latitudes for this time interval can be seen also in Fig. 2 (left panel).

As a whole a spectral amplitude of the $D$-component decreases towards low latitudes (Fig. 2, right panels) The amplitudes at conjugate stations MSR and BSV $(L \simeq 1.6)$ are very close, some variation between PTK and ADL $(L \simeq 2.1)$ is probably related to the local time difference $\left(\Delta \Phi \simeq 13^{\circ}\right)$ or to the coast effect. The amplitude spectrum of the $D$-component gradually decreases with frequency of $\sim f^{-\alpha}$, where $\alpha \simeq 1.5$ between $20-120 \mathrm{mHz}$. As the $D$-component should be only weakly influenced by resonant effects, it may
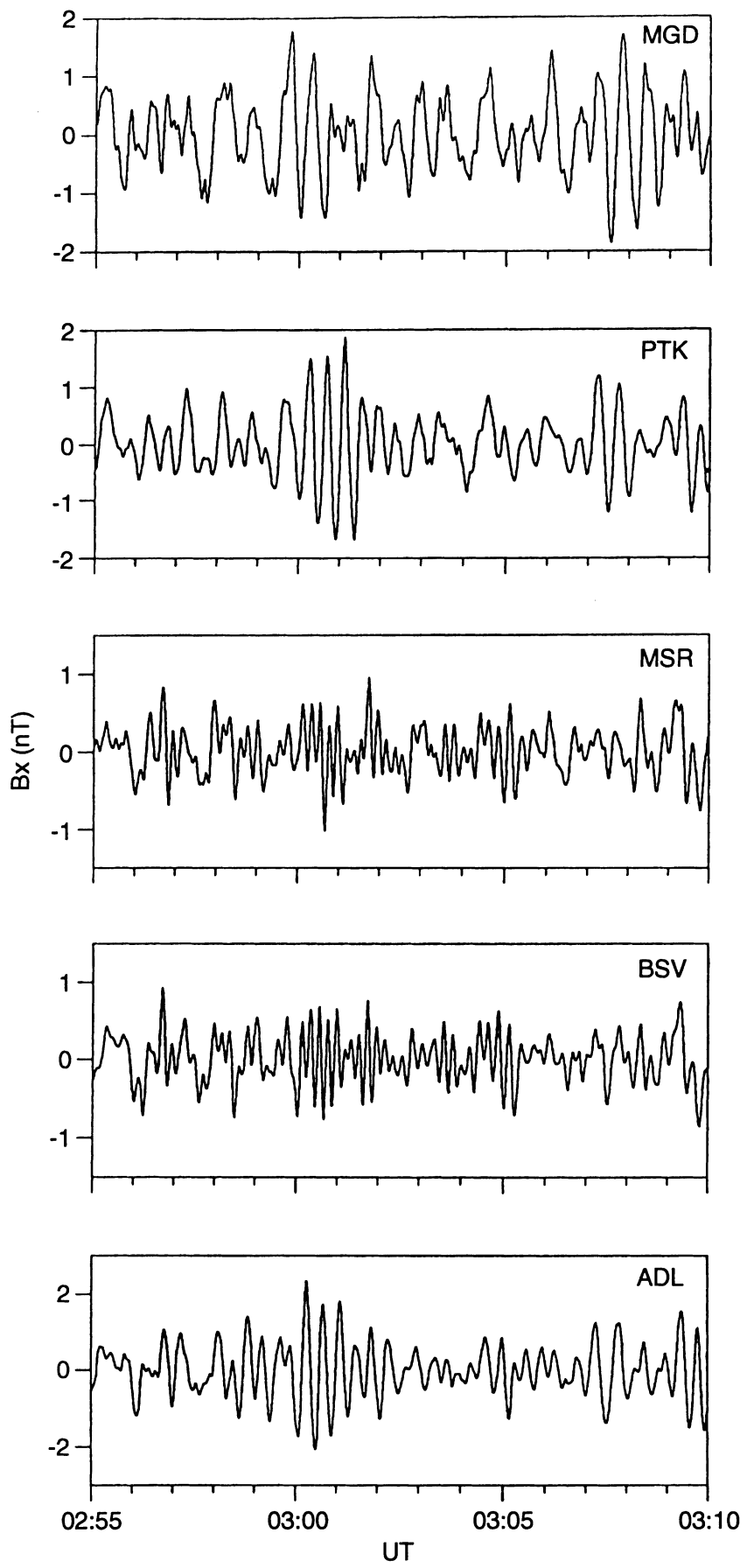

Fig. 1. Band-pass filtered in the range $10-150 \mathrm{mHz}$ magnetograms of $H$-component of pulsations, recorded on October 15, 1992, 02:5503:10 UT at mid-latitude stations of the $210^{\circ}$ meridional network (event 1)

roughly characterize the spectral content of an ULF pulsation source.

In order to discriminate between a source frequency and local resonance frequencies and to isolate more clearly the fine spectral structure of the $H$-component, the background slope of each spectral curve has been compensated by the multiplication of spectral amplitudes by the factor $f^{\alpha}$. This procedure is similar to the pre-whitening technique or to the subtraction of general 

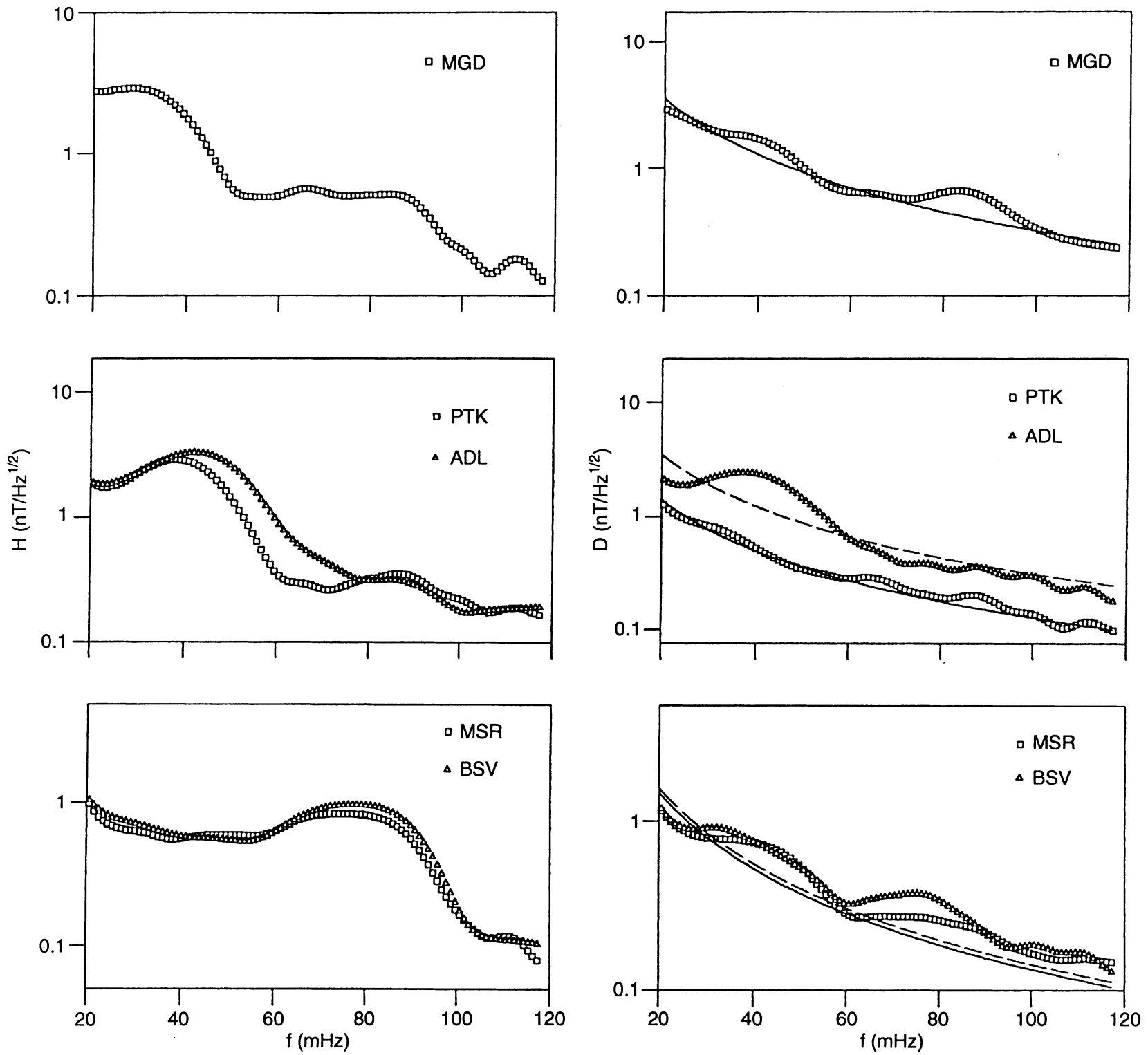

Fig. 2. Amplitude spectral densities for the interval 02:58-03:08 UT (event 1) of $H$-component (left panel) and of $D$-component (right panel). Spectral amplitudes are indicated by symbols, power best fit are shown by solid and dashed lines

spectral slopes, used by Vellante et al. (1993). After the elimination of the average spectral slope the fine structure of the $H$-component spectra exhibits specific pronounced spectral peaks (Fig. 3, left panel). Their frequencies systematically change with latitude: from $35 \mathrm{mHz}$ at $L=2.85$ (MGD) to $45-50 \mathrm{mHz}$ at $L=2.1$ (PTK/ADL), and further up to $85 \mathrm{mHz}$ at $L=1.6$ (MSR/BSV). Presumably, these frequencies correspond to local eigen frequencies of field-line Alfven oscillations. The additional peak at $\sim 85 \mathrm{mHz}$ at MGD corresponds to a higher harmonic. The method of extracting resonant effects, which uses the $H(f) / D(f)$ ratio (Baransky et al., 1995; Vellante et al., 1993), also stresses the same frequencies (Fig. 3, right panels). The additional argument in favor of the resonant nature of these spectral peaks is the fact that the signal coherency of $H$-component between conjugate stations PTK-ADL and MSR-BSV increases in the relevant frequency bands (Fig. 4). At the same time the non-resonant $D$-component at all frequencies (dashed line in Fig. 4) is poorly correlated between stations. Low coherency between ULF signals in the same frequency range does not permit us to estimate reliably the latitudinal profile of phase characteristics.

Events of such type clearly demonstrate local resonance effects, whereas a central frequency of a probable source cannot be simply distinguished from ULF spectra at a single station. It may be speculated that local noisy contributions dominate over a band-limited source spectrum. However, cross-spectral analysis reveals an enhanced coherence between $D$-components of ULF signals recorded at distant stations at frequency $\sim 40 \mathrm{mHz}$ (Fig. 5). According to the relationship between the expected upstream frequency and IMF 

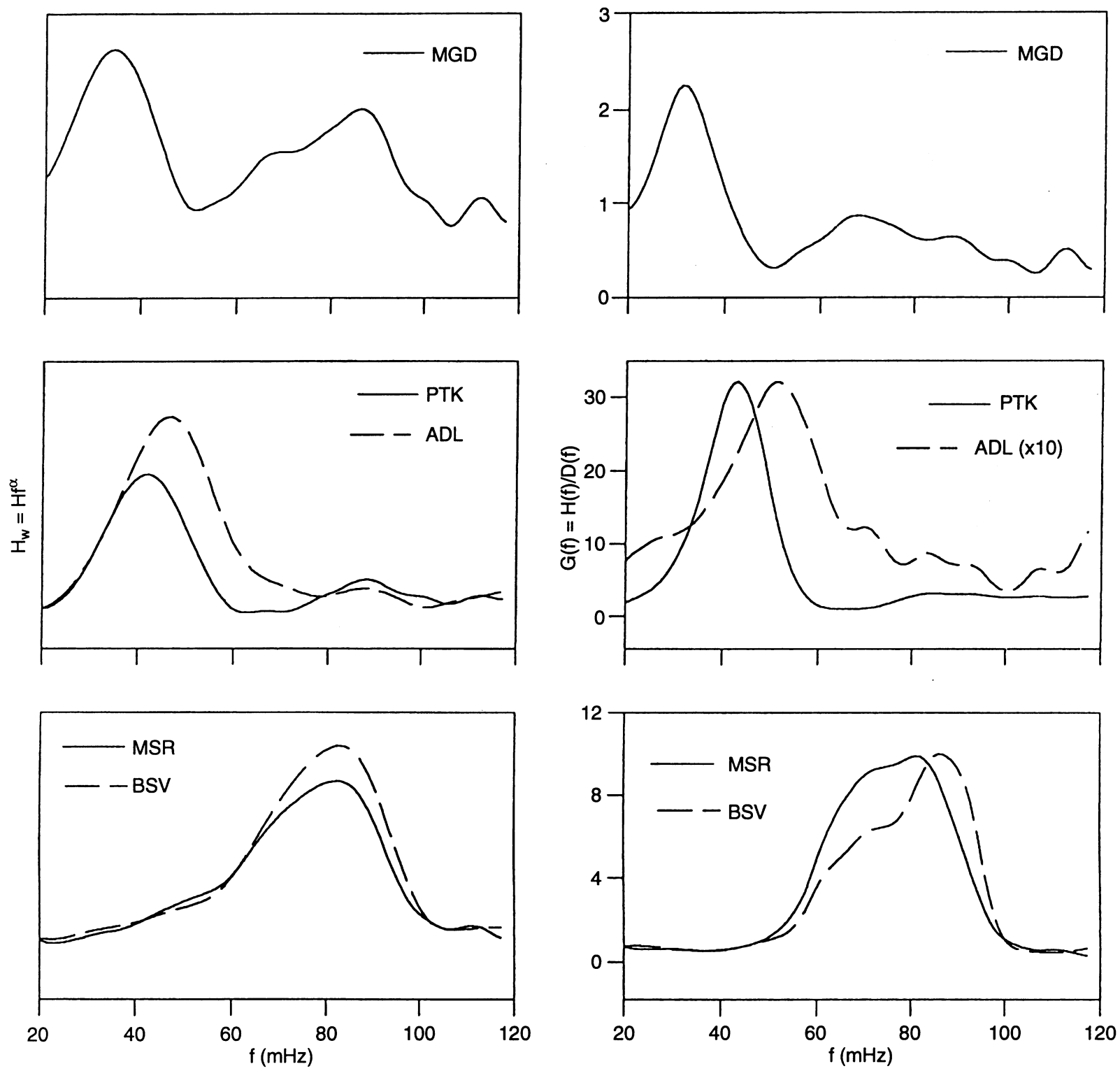

Fig. 3. Amplitude spectra of $H$-component (in arbitrary units) for the event 1 with corrected background slope $\left(H_{w}(f)=H(f) \cdot f^{\alpha}(l e f t\right.$ panel), and the $H(f) / D(f)$ spectral density ratio (right panel)

magnitude $f \simeq 6.3 B$ (Gugliel'mi, 1988) this frequency matches hour averaged (2-3 UT) IMF parameters: $B=6.3 \pm 4.1 \mathrm{nT}$.

\subsection{A source with a central frequency}

The principal feature of another ULF event (2) 21:5522:15 UT, 13.10 .92 (Fig. 6) is the enhanced amplitude spectral density at a certain frequency throughout the network. This event occurs also in a moderately disturbed geomagnetic situation: $K_{p}=4^{\circ}, D_{s t}=-30 \mathrm{nT}$.

In this event the $D$-component spectra have a common maximum at $\sim 60 \mathrm{mHz}$, more or less evident at all stations (Fig. 7, right panel). The presence of a global forced response at this frequency also clearly reveals itself in the enhanced signal's coherency between stations. In contrast to the previous event, for event 2 the signal's coherency between distant stations in the band $50-90 \mathrm{mHz}$ is rather high, $\gamma>0.5$ (Fig. 8). The central frequency $\sim 60 \mathrm{mHz}$ approximately agrees with the $f \simeq 6.3 B$ relationship for the frequency of upstream waves for hour-averaged (21-22 UT) IMF magnitude $B=10.7 \pm 6.8 \mathrm{nT}$.

The spectral content of $H$-component for this event is more complicated compared with event 1 , which is caused, probably, by a comparable mixture of forced and local resonant oscillations. The spectral maxima at the frequencies $\sim 60 \mathrm{mHz}$ at $L=2.85$ (MGD), as well as $\sim 35 \mathrm{mHz}$ and $80 \mathrm{mHz}$ at $L \approx 2.1$ (PTK-ADL) (Fig. 7, left panel), correspond to local resonant frequencies of fundamental Alfven mode and its harmonics. This assumption is supported by the $H(f) / D(f)$ ratio (Fig. 9). The phase relationships between signals at 

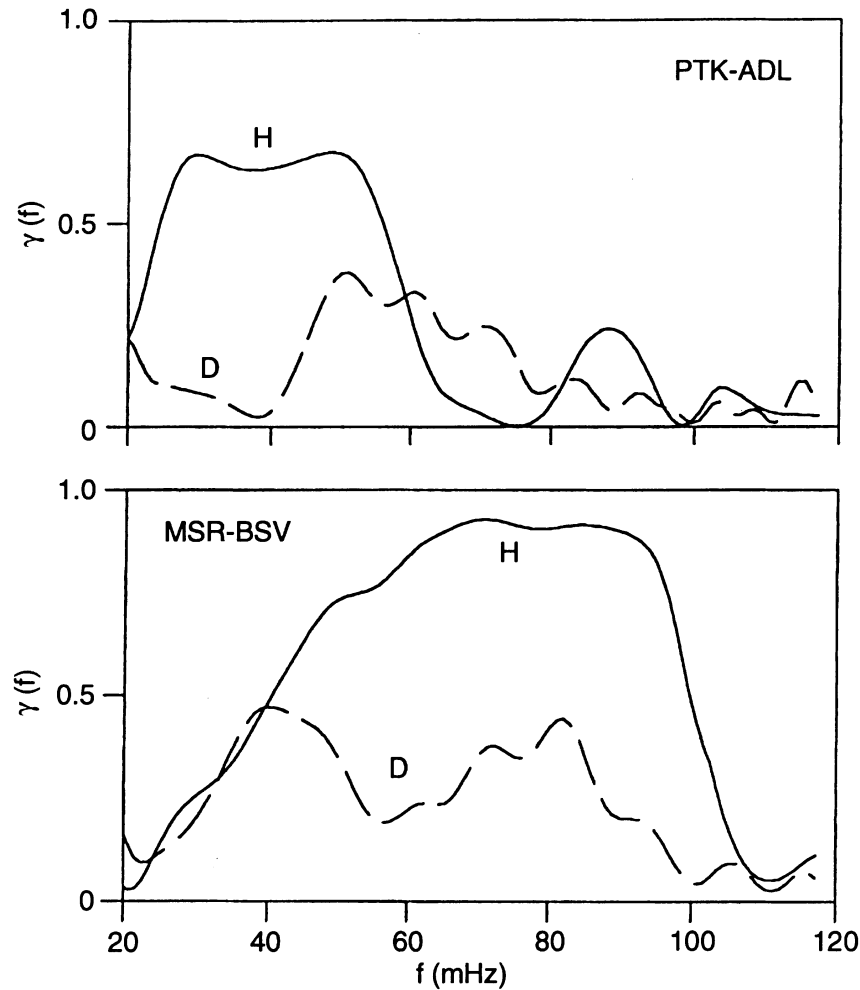

Fig. 4. Coherency coefficients $\gamma(f)$ for the event 1 for $H$ and $D$ components between the conjugate stations PTK-ADL $(L \approx 2.1)$ and $\operatorname{MSR}-B S V(L \approx 1.6)$

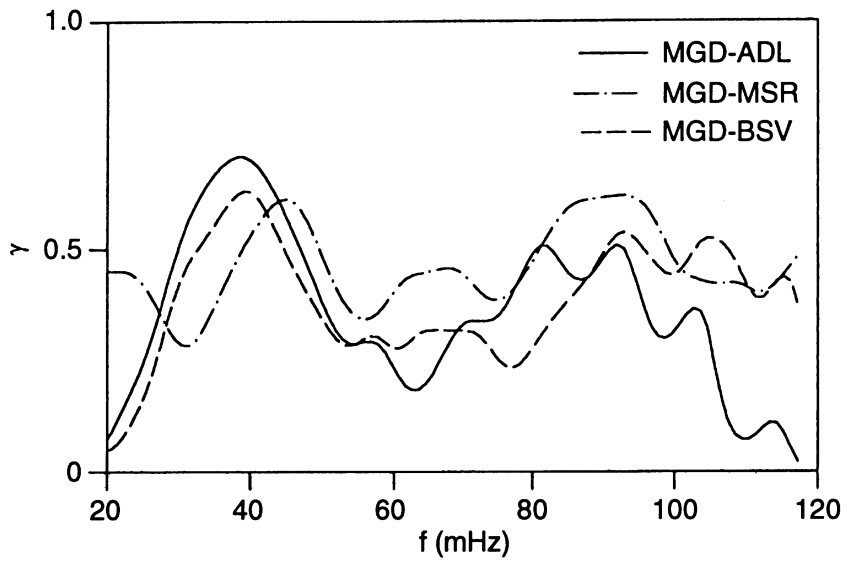

Fig. 5. Coherency coefficients $\gamma(f)$ for the event 1 for $D$ component between stations with different $L$-values

conjugate stations PTK-ADL (not shown) also confirm the existence of odd fundamental and even second harmonic: in the frequency bands of high coherency $(\gamma>0.5) H$-components are in-phase at low $(0-40 \mathrm{mHz})$ and out-of-phase at high $(70-95 \mathrm{mHz})$ frequencies.

The local nature of resonant oscillations at $L \approx 2.1$ results in low coherency between $H$-components at nearby stations PTK-MGD, PTK-MSR in the Northern Hemisphere and at ADL-BSV in the Southern Hemisphere near local resonant frequencies 35 and $80 \mathrm{mHz}$ (Fig. 10). At the same time a high coherency between ULF signals at stations from different latitudes is observed at a source frequency $\sim 65 \mathrm{mHz}$ (Fig. 10).
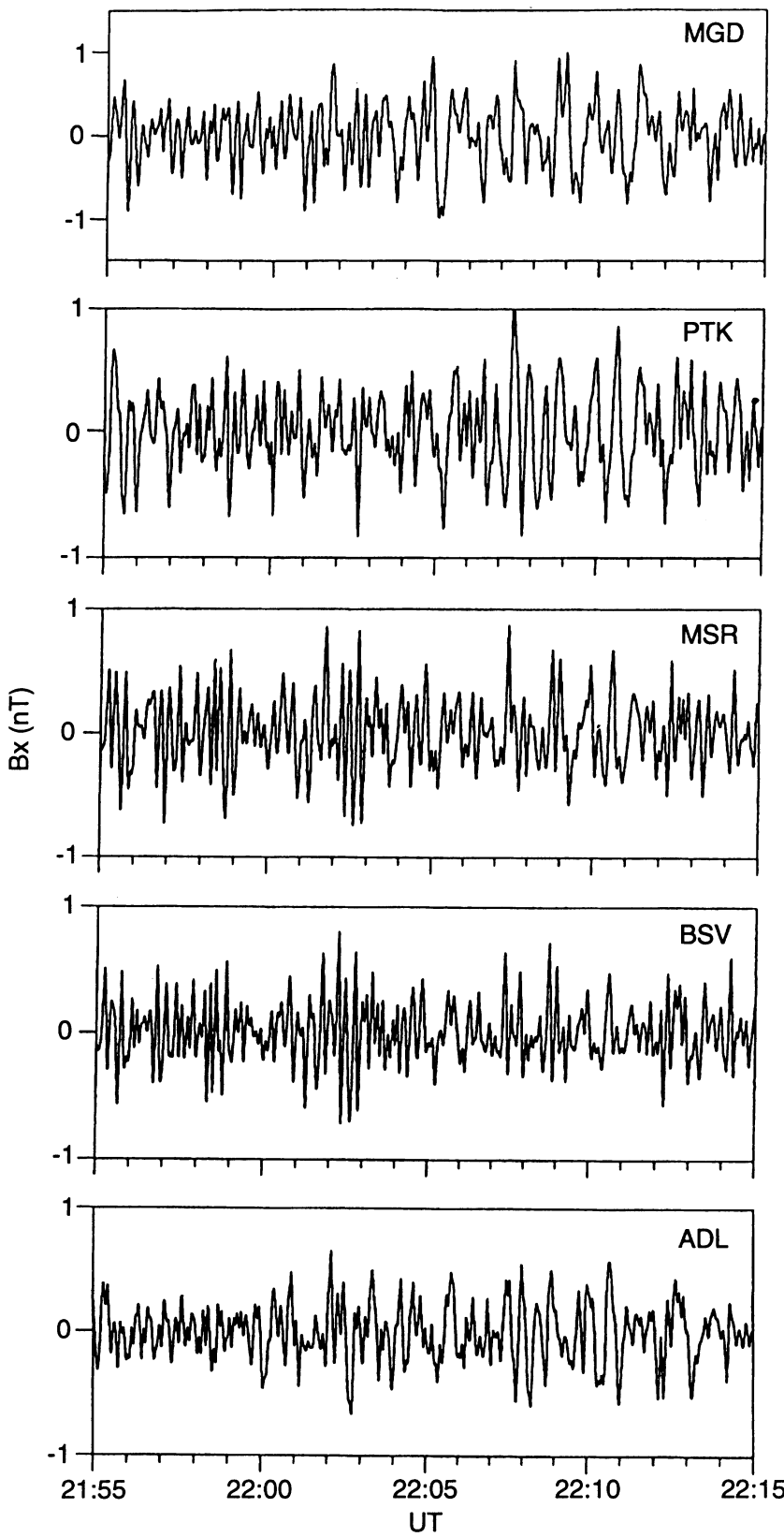

Fig. 6. Band-pass filtered in the range $10-150 \mathrm{mHz}$ magnetograms of $H$-component of pulsations, recorded on October 13, 1992, 21:5522:15 UT (event 2)

\subsection{Amplitude gap in the latitude-period distribution}

The spectral analysis of $H$-component reveals an interesting peculiarity of this class of ULF events. At the "common" frequency $\sim 60 \mathrm{mHz}$, which corresponds to a probable central frequency of an ULF source, a "gap" emerges at $L \approx 2.1$ in the meridional distribution of power spectral density (Fig. 7, left panel). The local minimum in the meridional distribution of pulsation's amplitudes in this frequency range has been observed at both conjugate stations PTK and ADL. Meanwhile, wide spectral maxima are observed at $L=2.85$ (MGD) and $L \approx 1.6(\mathrm{MSR} / \mathrm{BSV})$. Moreover, at this frequency at far magnetic shells (MGD-MSR and MGD-BSV) the 

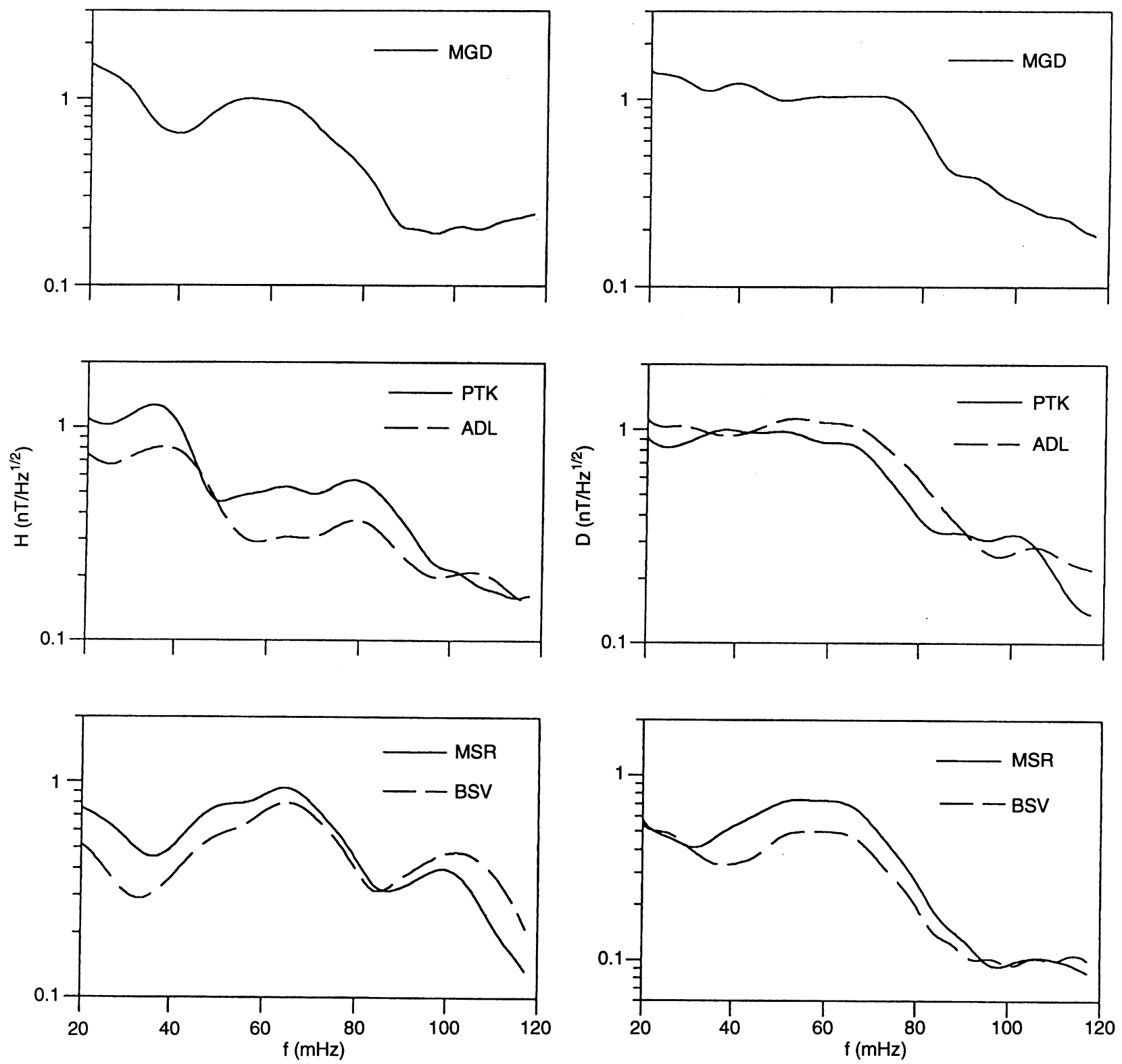

Fig. 7. Amplitude spectra of $H$ (left) and $D$ (right) components of pulsations for the event 2

$H$-components of signals in both hemispheres are even more coherent than the signals in closer shells (PTKMSR, MGD-PTK, MGD-ADL and ADL-BSV) (Fig. 10). AT both sides of a "gap" $H$-components of pulsations have no pronounced phase shift.

\subsection{Quasi-gradient method}

The key moment in the reconstruction of a meridional distribution of resonant frequencies is related to the interpretation of spectra observed at $L \approx 1.6$ (MSR/ BSV). Two frequencies, which are about $60 \mathrm{mHz}$ and $100 \mathrm{mHz}$, dominate the spectra of $H$-component (Fig. 7, bottom left plot). In principle, these peaks could be interpreted in two ways. On one hand, one may assume that $60 \mathrm{mHz}$ is a fundamental resonant frequency and $100 \mathrm{mHz}$ is its second harmonic. On the other had, one may believe that the high-frequency peak corresponds to a local fundamental frequency, while low-frequency peak is a non-resonant response. To resolve this ambiguity the standard gradient method (Baransky et al., 1995), which could indicate resonant frequencies, cannot be applied because of too large separations between stations. Nonetheless, some modification of the gradient method, in which the data of two nearly conjugated stations at $L \approx 1.6$ (MSR and BSV) are used, can be applied. The mismatch in longitude between them is about $0.1^{\circ}$ or $\Delta L=0.03$. Plots from the top to the bottom in Fig. 11 demonstrates the results of the quasi-gradient analysis:

a. Ratio $G(f)=H_{L}(f) / H_{H}(f)$ between spectral densities of $H$-components from the lower latitude station (BSV, $L=1.57$ ) and from the higher latitude station (MSR, $L=1.6$ ); 


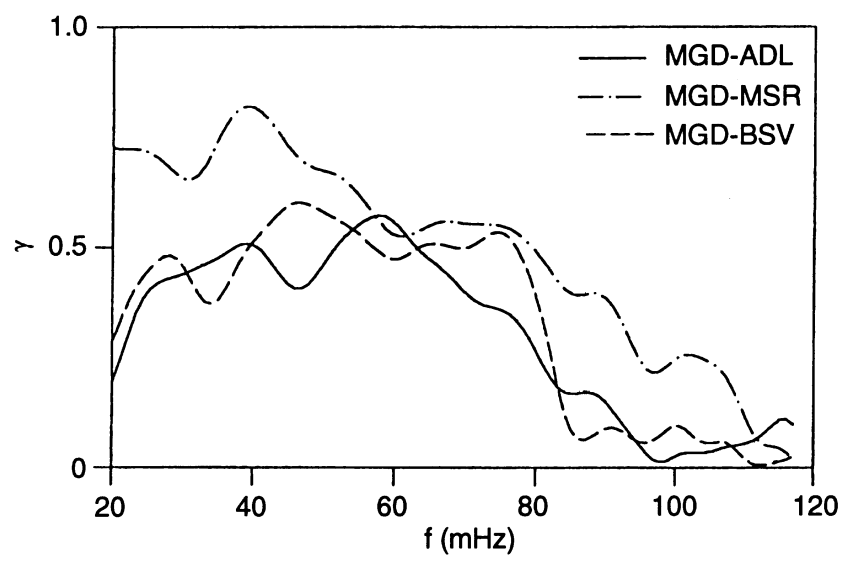

Fig. 8. Coherency coefficients $\gamma(f)$ for the event 2 for $D$ component between stations with different $L$-values

b. Phase difference $\Delta \varphi(f)(\Delta \varphi<0$ when the signal propagates towards higher $L$-values);

c. Coherency coefficient $\gamma(f)$.

The ULF signals at conjugate stations are highly coherent, $\gamma>0.85$, in wide frequency band 45-105 $\mathrm{mHz}$ (Fig. 11). The transition of $G(f)$ over 1 and the minimum value of $\Delta \varphi(f)$, both typical for any resonance region (Baransky et al., 1995), can be seen only near the high-frequency peak. Hence, the amplitude and phase gradients definitely indicate the high frequency peak as a resonant one, while low-frequency spectral enhancement has a non-resonant nature. This conclusion has been supported by the examination of $H(f) / D(f)$ ratio at each station, which also increased in the region of the high-frequency spectral peak (Fig. 9, bottom panel).

This interpretation can be verified also by the phase relationships between signals at conjugated stations: phase difference between $H$-components for two subsequent Alfven harmonics should be opposite. However, in reality $H$-components at BSV and MSR are in-phase in the whole frequency range $40-100 \mathrm{mHz}$ (Fig. 11).

\subsection{Statistical distribution}

Two examples shown are typical cases of possible frequency-latitude distributions. In one extreme case (type 2 events) spectral maxima are seen at the same frequency in both $H$ and $D$ components and the resonant effects are obscured. In contrast, in type 1 events, when a wide-band noisy component is prevailing, the local resonant responses are more evident.

Commonly, in the spectral content of any Pc3 event both forced oscillations produced by an external source and local Alfven distortions are present simultaneously. Discrimination between them is possible with the help of the polarization or the gradient analysis of a resonant component and the cross spectral analysis of a nonresonant component. Hereafter these approaches are verified on a statistical basis.
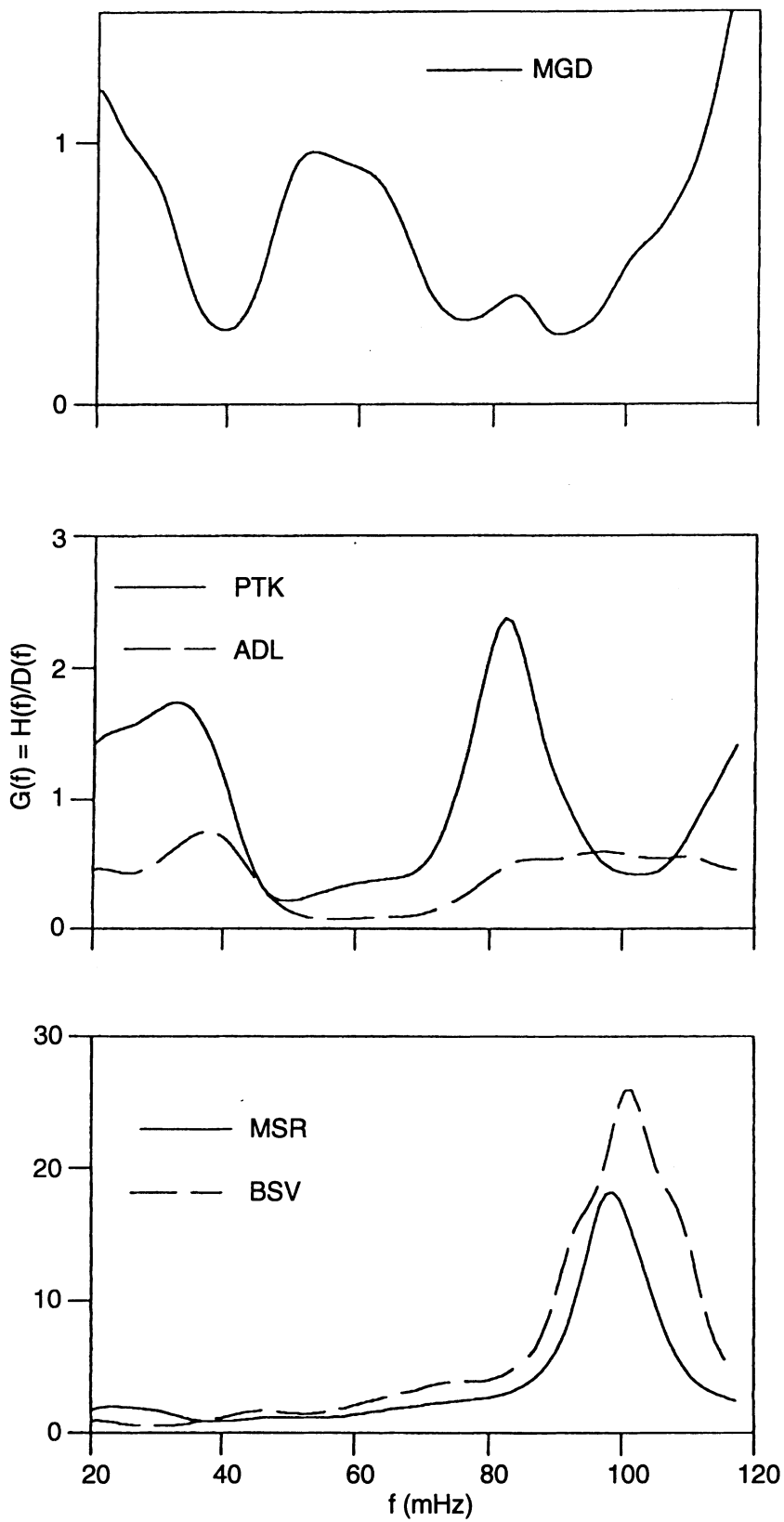

Fig. 9. The $H(f) / D(f)$ spectral density ratio for event 2

In total we have analyzed about $60 \mathrm{~h}$ of Pc3 activity, recorded in time intervals 05-15 LT (20-06 UT). A current frequency of spectral maxima has been estimated in a moving 15-min window for all the intervals with Pc3 activity at each station. The frequency of a local peak has been determined when in a slope-corrected spectrum a height of a peak $A_{\max }$ was above a certain threshold $A_{\mathrm{o}}$, i.e., $A_{\max }>A_{\mathrm{o}}$, whereas the value of $A_{\mathrm{o}}$ was gradually increased with latitude. The resultant statistical dependence of the number of events $N(f)$ with a spectral peak at frequency $f$ is presented as histograms in Fig. 12a. The statistical distributions of the $H$-component emphasize the most probable frequency of spectral maxima at $40-50 \mathrm{mHz}$ at all stations. The $N(f)$ for the $H / D$ ratio (Fig. 12b) demonstrates 

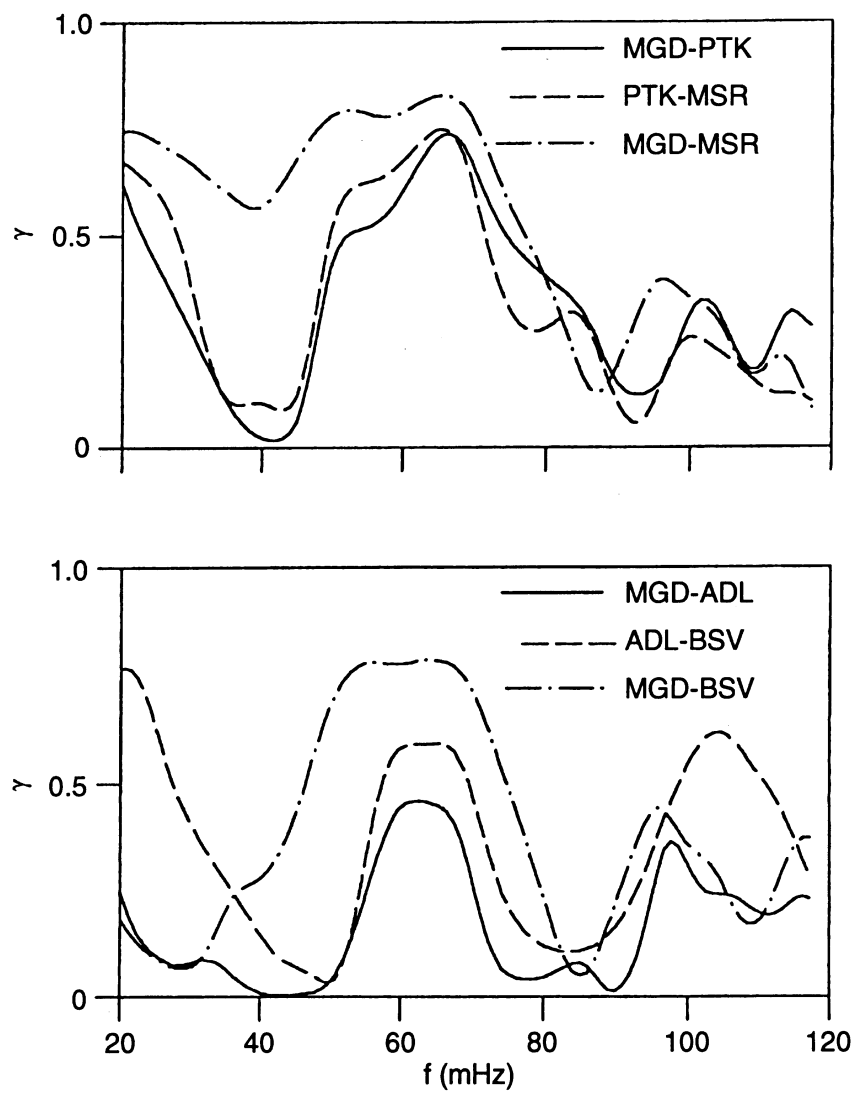

Fig. 10. Coherency coefficients $\gamma(f)$ of $H$-components between different pairs of stations MGD, PTK, MSR for event 2. The bottom panel is the same for the stations MGD, ADL, and BSV

enhancements at some particular frequencies for each station. It is natural to relate them to the local frequencies of resonant shell oscillations. These frequencies are $40-50 \mathrm{mHz}$ at $L=2.85,40-50 \mathrm{mHz}$ and $80-90$ $\mathrm{mHz}$ at $L \approx 2.1$ and $80-100 \mathrm{mHz}$ at $L \approx 1.6$.

The solar wind and IMF parameters are available only for limited intervals of the analyzed period. No obvious distinctions between these parameters, such as $B, B_{z}, V$, etc., between ULF events have been seen. However, the solar wind velocity $V \simeq 712 \pm 32 \mathrm{~km} / \mathrm{s}$ during the type 1 event was somewhat higher than in event 2: $V=624 \pm 21 \mathrm{~km} / \mathrm{s}$. In our opinion, the physical reason for the observed differences between those two types of events are related to small-scale features of the solar wind (turbulence level in the magnetosheath and its spectral form, intensity of upstream waves, etc.). All these features are not reflected in the 1-h averaged parameters from the IMF catalogue.

\subsection{Dynamic hydromagnetic spectroscopy}

In contrast to the problem of hydromagnetic diagnostics of magnetospheric plasma, for the study of correlative relationships between the IMF and ULF pulsations the monitoring of a central frequency of a source (which, most probably, is the dominant frequency of upstream waves) is more important. For that the preliminary
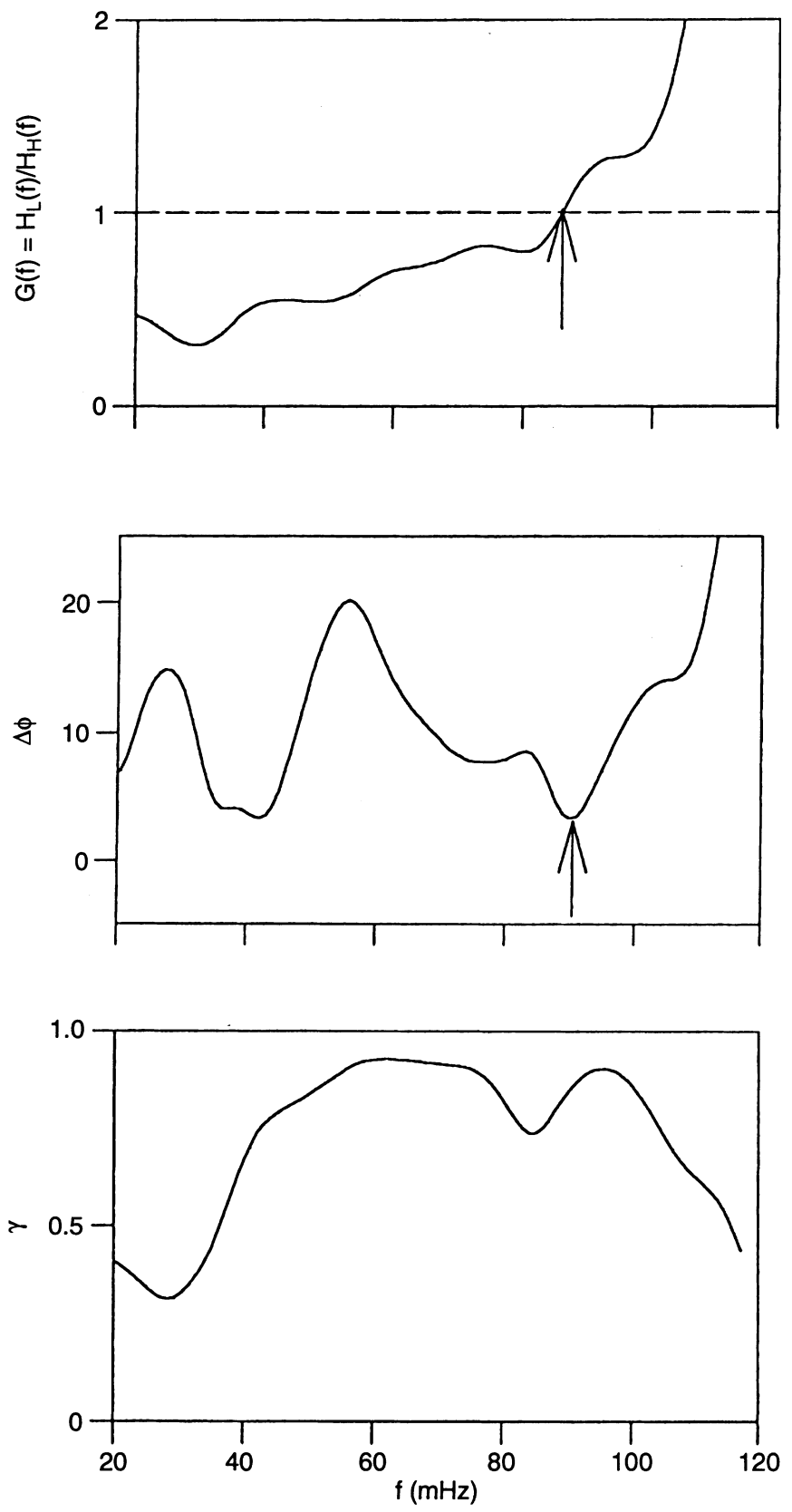

Fig. 11. The results of the quasi-gradient analysis of event 2 at nearconjugate stations MSR $(H)$ and $\operatorname{BSV}(L)$ (from top to bottom): spectra ratio $G(f)=H_{L}(f) / H_{H}(f)$; phase difference $\Delta \varphi(f)$ and coherency $\gamma(f)$. The resonant frequency of a field line between the stations is marked by an arrow

separation of different sources of ULF activity and the elimination of the resonant distortions of spectra are necessary. A rather simple technique may help to identify the nature of spectral peaks and to resolve different sources of the ULF pulsation's content. The principal possibility to monitor the temporal variations of both the local resonant frequency $f_{R}$ and the source frequency $f_{\mathrm{o}}$ is illustrated in Fig. 13. The $5 \mathrm{~h}$ interval analyzed (onset on 21:00 UT on October 15, 1992; $\left.K_{p}=5^{\circ}, D_{s t}=35 \mathrm{nT}\right)$ is characterized by a relatively high pulsation activity during the whole interval. The upper plot demonstrates the contour of the $H(f) / D(f)$ 

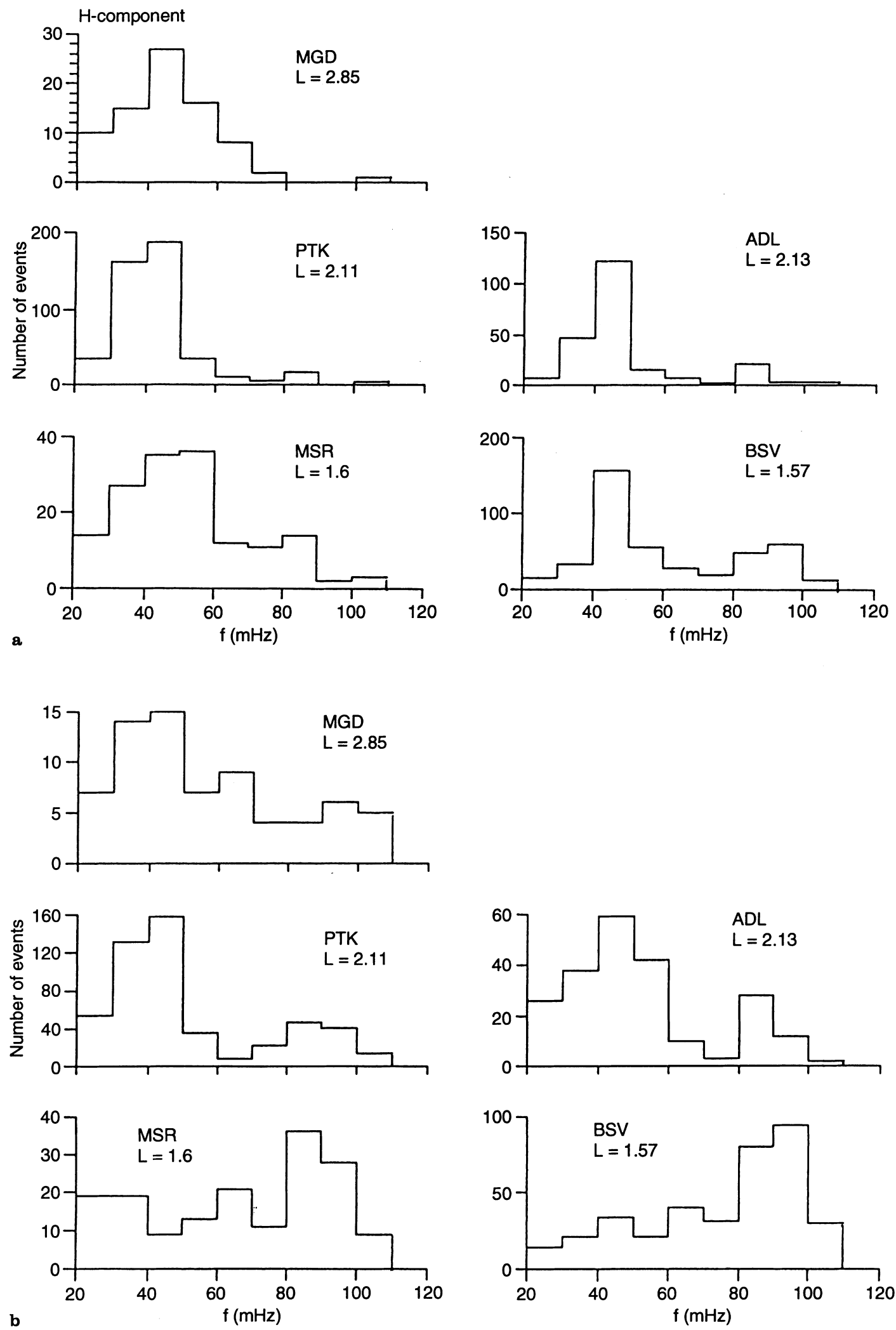

Fig. 12a,b. Histograms $N(f)$ of number of intervals with maxima of $H(f)$ (upper) and the $H(f) / D(f)$ ratio (lower) at a frequency $f$ for the Pc3 pulsations in September-October, 1992

ratio, estimated in a moving window, for the Pc3 series recorded at the station MSR. The lower plot presents, for the same time interval, the contours of the power cross-spectrum of $D$-components between distant stations MGD and MSR. Comparison of these two plots show that from the same ULF series both $f_{R} \simeq 80 \mathrm{mHz}$ 

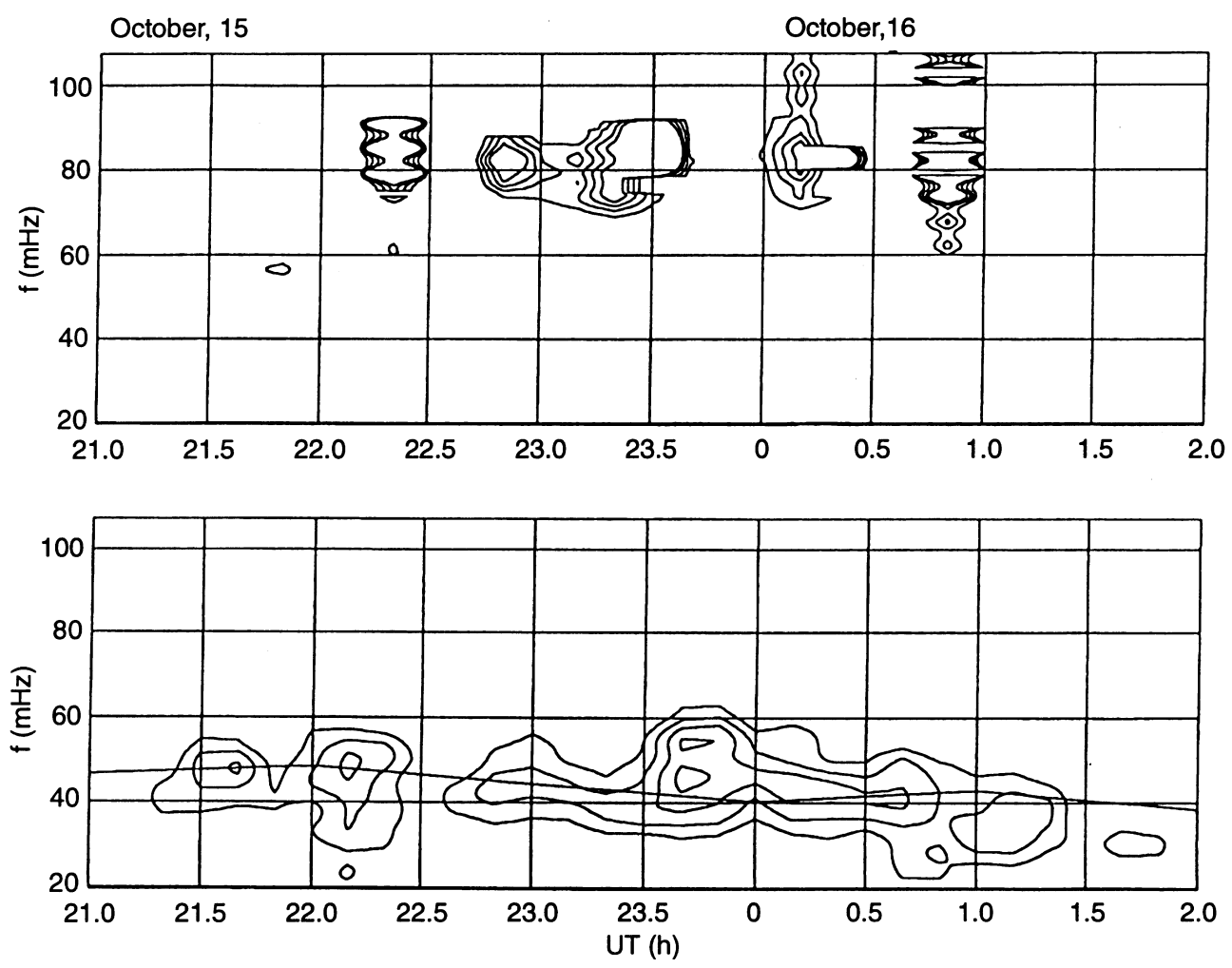

Fig. 13. Variations of the resonant frequency $f_{R}$ at $L=1.6$ (upper) and the expected source frequency $f_{\mathrm{O}}$ (bottom) of Pc3 pulsations recorded on October 15-16, 1992. The solid line in the lower panel shows the frequency estimated with the $f=6.3 \mathrm{~B}$ relationship

(determined as the frequency of maxima of $H(f) / D(f)$ ratio) and $f_{\mathrm{o}} \simeq 40-50 \mathrm{mHz}$ (determined as the frequency of amplitude cross-spectra maxima) can be extracted and monitored with good time resolution.

The probable upstream wave frequency estimated from the $f=6.3 B$ relationship varies between $48-40 \mathrm{mHz}$ and within the accuracy of the proposed technique coincides with the frequencies of the crossspectral maxima in Fig. 13. Successful attempts to separate these frequencies and to study their variations during the solar cycle have been undertaken recently by Vellante et al. (1995). It might be worth re-examining the numerous existing results on ULF-IMF correlations with a more correct preliminary analysis of ULF pulsation's spectra.

\section{Theoretical interpretation}

Specific features of the magnetosphere as an oscillatory system are caused by the presence in the eigen mode spectrum of the frequency bands with continuous spectra corresponding to the Alfven oscillations. An initial disturbance with frequency $f_{\mathrm{o}}$ generates two way processes in such systems: forced oscillation with a frequency $f_{\mathrm{o}}$ and transient oscillations with a spacevarying frequency $f_{R}(X)$. The variety of the possible wave regimes is due to the superposition of forced oscillations and transient oscillations, having an inhomogeneous spatial distribution of amplitudes and damping rates (Allan et al., 1986). Meridional distribution of a resultant oscillation at a given frequency $f_{\mathrm{o}}$ demonstrates rapid enhancement of amplitude and phase jump $(\sim \pi)$ in the Alfven resonance regions where $f_{\mathrm{o}} \approx f_{R}(L)$. Wave field structure near the resonant shell is described by the well-known steady-state solution from the fieldline resonance theory (Southwood, 1974). The observed amplitude-phase distribution of Pc3 along a meridian should be determined mainly by the relationships between source frequency $f_{\mathrm{o}}$ and local resonant frequencies of stations.

\subsection{Hydromagnetic diagnostics of magnetospheric plasma density}

The statistically determined values of local resonant frequencies $f_{R}(L)$ from ground-based data (Fig. 12b) have been compared with the eigen frequencies of Alfven oscillations in the inner plasmasphere. For that the numerical model of an Alfven resonator, which is based on the integration of local differential equation along a field line with boundary condition in the "thin ionospheric approximation", was used. This model can be applied till $L \simeq 1.4$, while at lower latitudes due to the mass loading effect of ionospheric ions the "thin ionosphere approximation" becomes invalid (Yumoto et al., 1995a; Pilipenko et al., 1997). The plasma distribution along a field line is characterized by the parameter $p, N(\theta)=N_{e q}(\cos \theta)^{-2 p}$, where $\theta$ is latitude. The fit between the frequencies of fundamental mode and its harmonics was obtained for the value $p=3.0$. The empirical whistler-ISEE model of Carpenter and Anderson (1992), being extrapolated into the region $L<2.2$, was used for the equatorial plasma density. The calculated values of frequencies of the first three 

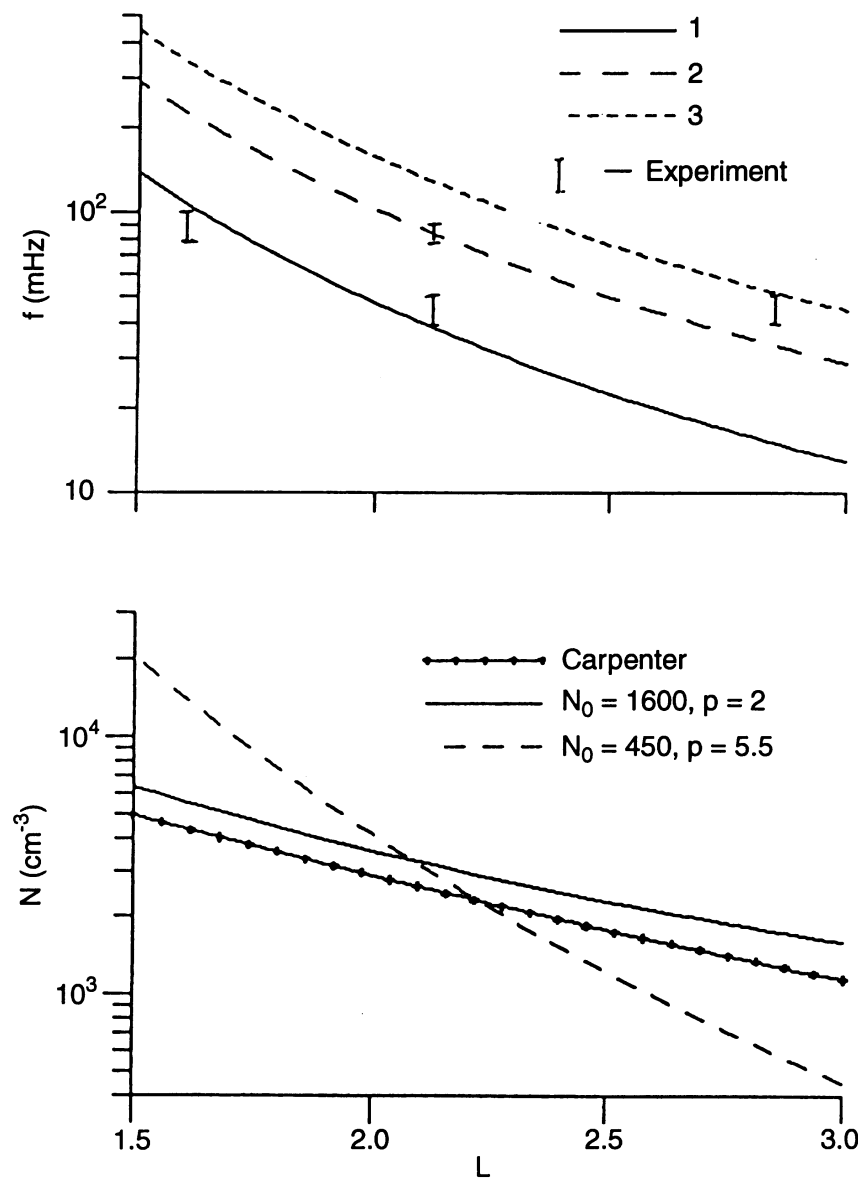

Fig. 14. Radial profile of latitude-dependent resonant frequencies approximating the experimental data (dashes) from the meridional network. Solid line corresponds to calculated frequencies of the fundamental mode, dashed lines - of 2-nd and 3-d harmonics

harmonics of Alfven oscillations along with the experimental values are plotted in Fig. 14. The $40-50 \mathrm{mHz}$ oscillations at $L=2.85$ may be interpreted as the third harmonic, $40-50$ and $80-90 \mathrm{mHz}$ at $L \approx 2.1$, as the fundamental mode and the second harmonic, and $80-100 \mathrm{mHz}$ at $L \approx 1.6$, as the fundamental mode of Alfven oscillations.

\subsection{Modeling of the gap in the meridional ULF structure}

In the second type of ULF events an effect of the local $H$-component amplitude gap, forming at $L \approx 2.1$, has been exhibited at both PTK and ADL. The effect of local decrease of the $H$-component amplitude at $L \approx 2.1$ cannot be explained by the local geoelectrical conditions (e.g., coast effect), because it was displayed at both conjugate stations. The emergence of the amplitude gap cannot be explained either by the presence near $L \approx 2.1$ of a cavity-mode node, because the necessary out-ofphase relationship between signals from both sides of a node does not hold. Next we consider the most probable, in our opinion, possibility of the interpretation of this effect.

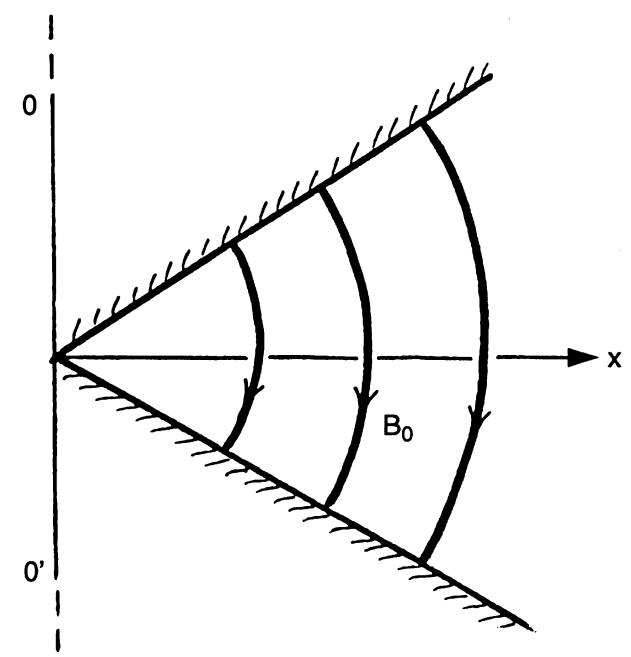

Fig. 15. A schematic plot of the geometry of the inner plasmasphere, used in the model calculation of ULF meridional structure

For modeling of spatial structure of ULF pulsations in the inner magnetosphere we numerically calculated the radial profiles of components of MHD wave excited by an external harmonic source. We used the magnetospheric model, whose meridional cross-section is a wedge, schematically shown in Fig. 15. The whole structure is formed by the azimuthal rotation of this cross section around the $00^{\prime}$ axis. Though this model is mathematically more complicated (variables are not separable) than the well-known "box-field" model (Southwood, 1974) or the "cylindrical wedge" model (Allan et al., 1986), it allows us to consider the decrease of the effective azimuthal wave number $k_{y}=m / L R_{E}$ and the inhomogeneity scale in the inner plasmasphere. Figure 16 displays the results of calculations of amplitudes (Fig. 16a) and phases (Fig. 16b) of the field aligned $B_{Z}$ (dashed line) and the azimuthal $B_{y}$ (solid line, corresponding to the resonant $H$-component at the Earth's surface) magnetic components. The following wave parameters have been adopted: azimuthal wave number $m=4$, source frequency $f_{\mathrm{o}}=50 \mathrm{mHz}$. The numerical results prove that a gap in a meridional profile of ULF wave amplitude can be formed on the source side of a resonant region. The phase jump by $\sim \pi / 2$ of $B_{Z}$-component (Fig. 16b) can be interpreted as the superposition in the magnetosphere of an "incident" wave from a source and a wave "reflected" with phase shift $\sim \pi / 2$ from a resonant magnetic shell. In the wave structure which is formed in front of a resonant maximum the local decrease of amplitude and the partial phase shift compensation take place. Thus, a situation is possible like that shown in Fig. 16, when a comparison of ULF signals at several widely separated stations (marked by arrows) would display the gap at the mid-station and only a small phase shift $\left(\sim 60^{\circ}\right)$ between the stations at both sides of a resonant field line. At $210^{\circ} \mathrm{MM}$ network the non-monotonic amplitude profile emerges due to the resonant amplification of pulsations somewhere between $L=1.6$ and $L=2.1$. 


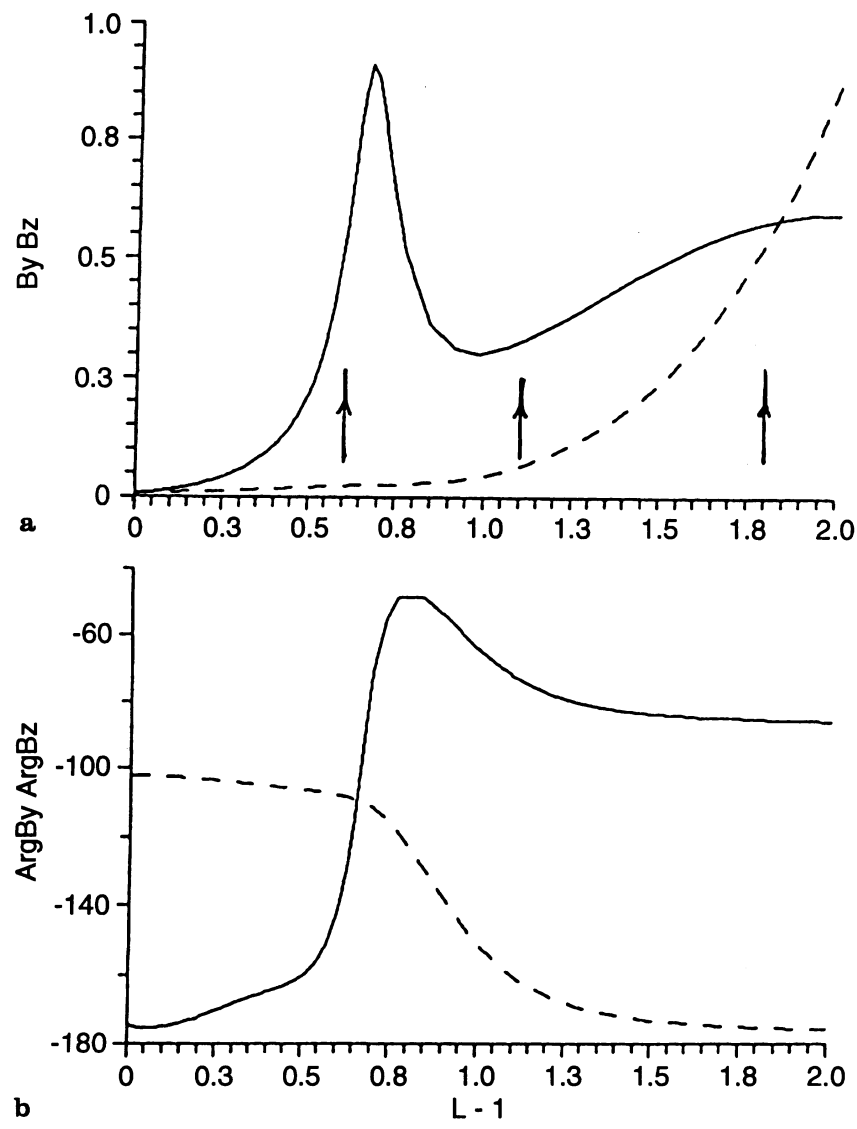

Fig. 16a,b. Numerically calculated radial distribution of the amplitudes $B_{y}, B_{z}$ (upper plots) and phases $\operatorname{Arg}\left(B_{y}\right), \operatorname{Arg}\left(B_{z}\right)$ (bottom plots) of the components of ULF wave structure in the magnetosphere. Wave parameters are $m=4, f=50 \mathrm{mHz}$

\section{Discussion and conclusion}

Analysis of the data of mid-latitude stations from the $210^{\circ} \mathrm{MM}$ network showed that spectral content of ULF pulsations at various latitudes may vary considerably from one station to another even at separations of $\Delta \Phi \simeq 7-8^{\circ}(\Delta L \simeq 0.5)$. The form of the observed spectra are mainly determined by features of the pulsation's source spectrum and by local response of the magnetospheric resonator. The identification of nature of spectral peaks, which was named the hydromagnetic spectroscopy, cannot be performed by the analysis of spectra of one component only, at some particular station. In an optimal way, this problem can be resolved by the combination of stations with small $(\sim 100$ $200 \mathrm{~km})$ and large $\left(\sim 10^{3} \mathrm{~km}\right)$ baselines. For the meridional network $210^{\circ} \mathrm{MM}$ the unambiguous isolation of resonant frequencies at $L \simeq 1.6$ was performed with the help of the quasi-gradient method, which uses the data of nearly conjugate stations. The results of the suggested method are in accordance with the results of the polarization method, based on the asymmetry of the spectra of two horizontal components. Using the experimentally measured set of resonant frequencies the magnetospheric plasma distribution has been restored both in a radial direction and along a field line. It is essential that the ULF pulsation recordings prove to be a reliable method of ground-based diagnostics of the magnetospheric plasma density at low latitudes also, where the standard whistler diagnostics becomes ineffective.

The numerical modeling of MHD wave structure proved that a gap in a meridional profile of ULF wave amplitude can be formed on the source side of a resonant region in the inner magnetosphere. In the gap, the amplitude decreases and the partial compensation of the resonant phase jump takes place. During observations at widely separated stations along a meridian (e.g., at $210^{\circ} \mathrm{MM}$ profile) this effect may obscure a phase jump at a resonant shell.

In some cases (type 1 events) the level of ULF disturbances, transported into the inner magnetosphere by large-scale compressional modes, is below the magnetospheric noise. The appearance of quasi-sinusoidal signals at the ground is caused mainly by magnetospheric resonances. However, a frequency band of a source can be found by the coherency analysis of ULF signals from meridionally separated stations.

In a prevailing number of events (type 2) the source spectrum is identified as an enhanced spectral power density at certain frequency. This frequency might be related to the frequency of unstable upstream waves (Yumoto, 1985) or to the selective penetration of solar wind energy into the magnetosphere (Pilipenko, 1990). Simple methods of hydromagnetic spectroscopy enable us to monitor simultaneously from ULF data both the magnitude of IMF and plasma density in the magnetosphere.

Acknowledgements. The authors express their sincere thanks to all members of the $210 \mathrm{MM}$ Magnetic Observations Project for their ceaseless support and for the use of the database, especially, to $\mathrm{S}$. Solovyiev (IKFIA), E. Vershinin and A. Buzevich (IKIR), who are conducting experimental field observations in Siberia and Kamchatka. We appreciate the helpful criticism of both referees. This research was partly supported by grant 97-05-65404 from Russian Fund for Fundamental Research.

Topical Editor K.-H. Glassmeier thanks U. Villante and S. Fujita for their help in evaluating this paper.

\section{References}

Allan, W., S. P. White, and E. M. Poulter, Impulse-excited hydromagnetic cavity and field line resonances in the magnetosphere, Planet. Space. Sci., 34, 371-385, 1986.

Baransky, L. N., E. N. Fedorov, N. A. Kurneva, V. A. Pilipenko, A. W. Green, and E. W. Worthington, Gradient and polarization methods of the ground-based hydromagnetic monitoring of magnetospheric plasma, J. Geomagn. Geolect., 47, 1293-1309, 1995.

Carpenter, D. L., and R. R. Anderson, An ISEE/Whistler model of equatorial electron density in the magnetosphere, J. Geophys. Res., 97A, 1097-1108, 1992.

Green, A. W., E. W. Worthington, L. N. Baransky, E. N. Fedorov, N. A. Kurneva, V. A. Pilipenko, D. N. Shvetzov, A. A. Bektemirov, and G. V. Philipov, Alfven field line resonances at low latitudes $(L=1.5)$, J. Geophys. Res., 98A, 15693-15699, 1993.

Gugliel'mi, A. V., The coefficient of relationship between the Pc3 frequency with IMF, Geomagn. Aeron. 28, 465-468, 1988. 
Kurchashov, Y. P., Y. S. Nikomarov, V. A. Pilipenko, and A. Best, Field-line resonance effects in a local meridional structure of mid-latitude geomagnetic pulsations, Annales Geophysicae, 5A, 147-154, 1987.

Odera, T. J., Solar wind controlled pulsations, a review, Rev. Geophys. 24, 55-74, 1986.

Pilipenko, V. A., ULF waves on the ground and in space, J. Atmos. Terr. Phys., 52, 1193-1209, 1990.

Pilipenko, V., K. Yumoto, E. Fedorov, N. Kurneva, and F. Menk, Field line Alfven oscillations at low latitudes, Mem. Kyushu Univ. Ser D, 30, 23-43, 1997.

Russel, C. T., and B. K. Fleming, Magnetic pulsations as a probe of the interplanetary magnetic field: a test of Borok B index, J. Geophys. Res., 81, 5882-5886, 1976.

Southwood, D. J., Some features of field line resonances in the magnetosphere, Planet. Space, Sci., 22, 483-491, 1974.

Vellante, M., U. Villante, M. De Lauretis, R. Core, A. Best, D. Lenners, and V. A. Pilipenko, Simultaneous geomagnetic pulsa- tion observations at two latitudes: resonant mode characteristics, Annales Geophysicae, 11, 734-741, 1993.

Vellante, M., U. Villante, M. De Lauretis, and G. Barchi, Solar cycle variation of the dominant frequencies of Pc3 geomagnetic pulsations at $L=1.6$, Geophys. Res. Lett., 23, 1505-1508, 1995.

Webb, D. C., L. J. Lanzerotti, and C. G. Park, A comparison of ULF and VLF measurements of magnetospheric cold plasma densities, J. Geophys. Res., 82, 5063-5072, 1977.

Yumoto, K., Low-frequency upstream wave as a probable source of low-latitude Pc3-4 magnetic pulsations, Planet. Space Sci., 33, 239-249, 1985.

Yumoto, K., V. A. Pilipenko, E. N. Fedorov, N. A. Kurneva, and K. Shiokawa, The mechanisms of damping of geomagnetic pulsations, J. Geomagn. Geoelectr., 47, 163-176, 1995a.

Yumoto, K., and the 210 MM Magnetic Observation Group, Initial results from the 210 Magnetic meridian project - review, J. Geomagn. Geoelectr., 47, 1197-1213, 1995 b. 